\title{
Preclinical Evaluation of a Radioiodinated Fully Human Antibody for In Vivo Imaging of Vascular Adhesion Protein-1-Positive Vasculature in Inflammation
}

\author{
Anu Autio ${ }^{1}$, Petri J. Vainio ${ }^{2,3}$, Sami Suilamo ${ }^{1}$, Antti Mali ${ }^{4}$, Jani Vainio ${ }^{2}$, Tiina Saanijoki ${ }^{1}$, Tommi Noponen ${ }^{5}$, \\ Helena Ahtinen ${ }^{1}$, Pauliina Luoto ${ }^{1}$, Mika Teräs ${ }^{1}$, Sirpa Jalkanen ${ }^{6}$, and Anne Roivainen ${ }^{1,7}$ \\ ${ }^{1}$ Turku PET Centre, University of Turku and Turku University Hospital, Turku, Finland; ${ }^{2}$ Biotie Therapies Corporation, Turku, \\ Finland; ${ }^{3}$ Clinical Pharmacology Unit, Turku University Hospital and University of Turku, Turku, Finland; ${ }^{4}$ MAP Medical \\ Technologies Oy, Tikkakoski, Finland; ${ }^{5}$ Department of Nuclear Medicine, Turku University Hospital, Turku, Finland; ${ }^{6}$ MediCity \\ Research Laboratory, University of Turku, Turku, Finland; and ${ }^{7}$ Turku Center for Disease Modeling, University of Turku, Turku, Finland
}

\begin{abstract}
Vascular adhesion protein-1 (VAP-1) is an endothelial glycoprotein mediating leukocyte trafficking from blood to sites of inflammation. $\mathrm{BTT}-1023$ is a fully human monoclonal anti-VAP-1 antibody developed to treat inflammatory diseases. In this study, we preclinically evaluated radioiodinated BTT-1023 for inflammation imaging. Methods: Rabbits were intravenously injected with radioiodinated BTT-1023. Distribution and pharmacokinetics were assessed by PET/CT up to $72 \mathrm{~h}$ after injection. Human radiation dose estimates for ${ }^{124}$ I-BTT-1023 were extrapolated. Additionally, rabbits with chemically induced synovitis were imaged with ${ }^{123}$ I-BTT-1023 SPECT/CT. Results: Radioiodinated BTT-1023 cleared rapidly from blood circulation and distributed to liver and thyroid. Inflamed joints were delineated by SPECT/CT. The estimated human effective dose due to ${ }^{124} \mathrm{I}-\mathrm{BTT}-1023$ was $0.55 \mathrm{mSv} / \mathrm{MBq}$, if blockage of thyroid uptake is assumed. Conclusion: The radioiodinated BTT1023 was able to detect mild inflammation in vivo. Clinical ${ }^{124}$ I-BTT$1023 \mathrm{PET}$ studies with injected radioactivity of $0.5-0.7 \mathrm{MBq} / \mathrm{kg}$ may be justified.
\end{abstract}

Key Words: antibody; radioiodination; nuclear imaging; rabbit; vascular adhesion protein-1

J Nucl Med 2013; 54:1315-1319

DOI: 10.2967/jnumed.113.120295

\section{$\mathbf{V}$} ascular adhesion protein-1 (VAP-1) is an endothelial glycoprotein that plays a key role in cellular trafficking by recruiting leukocytes from blood into lymphoid organs and inflamed tissues (1-3). VAP-1 is practically absent from the endothelial surface of normal tissues, but on inflammation, it is translocated from intracellular storage granules to the endothelial cell surface (2). For instance, dermal blood vessels in various inflammatory skin diseases (e.g., psoriasis) and synovial blood vessels in inflamed joints (e.g., rheumatoid arthritis) express VAP-1 on their surface (4-7). Besides being an adhesion molecule, VAP-1 also is

Received Jan. 23, 2013; revision accepted Mar. 20, 2013.

For correspondence contact: Anne Roivainen, Turku PET Centre, Kiinamyllynkatu 4-8, Fl-20521 Turku, Finland.

E-mail: anne.roivainen@utu.fi

Published online Jul. 11, 2013.

COPYRIGHT (c) 2013 by the Society of Nuclear Medicine and Molecular Imaging, Inc. a semicarbazide-sensitive amine oxidase. The end products of the reaction are highly potent inflammatory mediators. Blocking of VAP-1 reduces leukocyte adhesion, suggesting that VAP-1 is a potential target for antiinflammatory therapy (8).

PET with monoclonal antibodies, immuno-PET, can be applied to quantify expression of accessible antigens in the target tissue. Potentially, this specific application of PET could help in stratifying patients for antibody-based therapies. Another important application of immuno-PET could be its use in the development of antibodies as drugs. PET, using therapeutic antibodies labeled with, for example, ${ }^{124} \mathrm{I}$ or ${ }^{89} \mathrm{Zr}$, may provide useful quantitative information about pharmacokinetics, accumulation in targeted and nontargeted tissues, and saturation of the target antigen in patients. Immuno-PET may assist in accurate dosing of novel biologicals, possibly speeding the development of therapeutic antibodies, their fragments, or fusion proteins (9). Imaging agents targeting VAP-1 might be valuable not only for the diagnosis and planning of treatment in patients but also for in vivo imaging of leukocyte trafficking at sites of inflammation.

BTT-1023 is a recombinant fully human monoclonal antibody directed to human VAP-1. It belongs to the IgG4 class and carries intentional mutations to remove unfavorable Fc receptor binding properties and to stabilize the dimer (10). BTT-1023 binds to human VAP-1, thereby blocking its function as an adhesion molecule. It also recognizes VAP-1 of other primates and rabbits but does not cross-react with rat or mouse. BTT-1023 is potentially useful for the treatment and in vivo imaging of inflammatory conditions such as rheumatoid arthritis.

The purpose of this study was to obtain preclinical information on the whole-body distribution, pharmacokinetics, and inflammation detection of intravenously administered radioiodinated BTT-1023 in rabbits. For potential clinical use, human radiation doses of ${ }^{124}$ I-BTT-1023 were estimated from the rabbit data.

\section{MATERIALS AND METHODS}

Additional details on the methods are provided as supplemental data (available at http://jnm.snmjournals.org).

\section{Radioiodination and VAP-1 Binding of BTT-1023}

BTT-1023 was radioiodinated using the chloramine-T method followed by purification with size-exclusion chromatography. Radiochemical purity was analyzed using thin-layer chromatography. 
Binding of radioiodinated BTT-1023 to VAP-1 was evaluated using human VAP-1-transfected cells versus mock-transfected cells and radioimmunoassay or flow cytometry.

\section{Animals}

Selection of rabbits as the experimental animal was based on BTT1023 cross-reactivity. All studies were conducted with approval from the Lab-Animal Care and Use Committee of the State Provincial Office of Southern Finland and in compliance with Finnish laws relating to the conduct of animal experimentation. In total, 14 rabbits $(2.4-4.0 \mathrm{~kg})$ were used in the studies. For imaging, the rabbits were sedated and anesthetized. To induce synovitis, 4 rabbits were intraarticularly injected with phytohemagglutinin (11).

\section{PET Studies}

${ }^{124}$ I-BTT-1023 (52 $\left.\pm 2.5 \mathrm{MBq}\right)$ was injected intravenously, and whole-body PET/CT scanning was performed over the first $2 \mathrm{~h}$ and at 24,48 , and $72 \mathrm{~h}$ after injection (Discovery VCT; GE Healthcare). The radioactivity concentration in various organs was analyzed, and the results were expressed as standardized uptake values. During PET, serial blood samples were collected. The amount of intact ${ }^{124}$ I-BTT1023 as a function of time after injection was used for the calculation of pharmacokinetic parameters. After the last PET/CT scan, that is, $72 \mathrm{~h}$ after injection, various organs were excised, weighed, and measured for radioactivity using a $\gamma$-counter.

Distribution of radioactivity in several organs was also studied by autoradiography of cryosections. After autoradiography, adjacent sections were stained with anti-VAP-1 antibody and with hematoxylin-eosin.

Human radiation dose estimates were extrapolated from the rabbit data using OLINDA/EXM software.

\section{SPECT/CT}

${ }^{123}$ I-BTT-1023 (22 $\left.\pm 9.3 \mathrm{MBq}\right)$ was injected intravenously, and SPECT/CT scanning was performed at $2 \mathrm{~h}$ after injection (Symbia
TruePoint; Siemens Healthcare). The radioactivity concentration in the inflamed knee, the contralateral knee, and the contralateral muscle was analyzed.

\section{Statistics}

All results are expressed as mean \pm SD. Statistical analyses were performed with the Student $t$ test and linear regression analysis. A $P$ value of less than 0.05 was considered statistically significant.

\section{RESULTS}

\section{Inflammation Model}

Lymphocyte infiltrations are characteristic of inflamed human synovium, such as in rheumatoid arthritis (Fig. 1A). A rabbit model of acute synovial inflammation was generated using chemical induction. After phytohemagglutinin challenge, all rabbits had swollen and warm joints as symptoms of inflammation. Closer histologic examination revealed only mild inflammation, with occasional granulocytes in the inflamed knee in comparison to the control knee (Figs. 1B and 1C). Unlike the human inflamed synovium, which has abundant expression of VAP-1 on the vasculature (Fig. 1D), VAP-1 induction was minor in this acute inflammatory state (Fig. 1E). Unexpectedly, there were also VAP-1-positive vessels in the healthy contralateral side (Fig. 1F). This finding could have been due to a systemic response to phytohemagglutinin injected in the knee cavity or VAP-1 expressed constitutively in the joint vasculature of the rabbit.

\section{VAP-1 Binding of Radioiodinated BTT-1023}

According to radioimmunoassay, binding of ${ }^{124}$ I-BTT-1023 to human VAP-1-transfected cells was approximately 600-fold higher than binding to mock-transfected cells, and binding was eliminated in competition assays using an excess of unlabeled
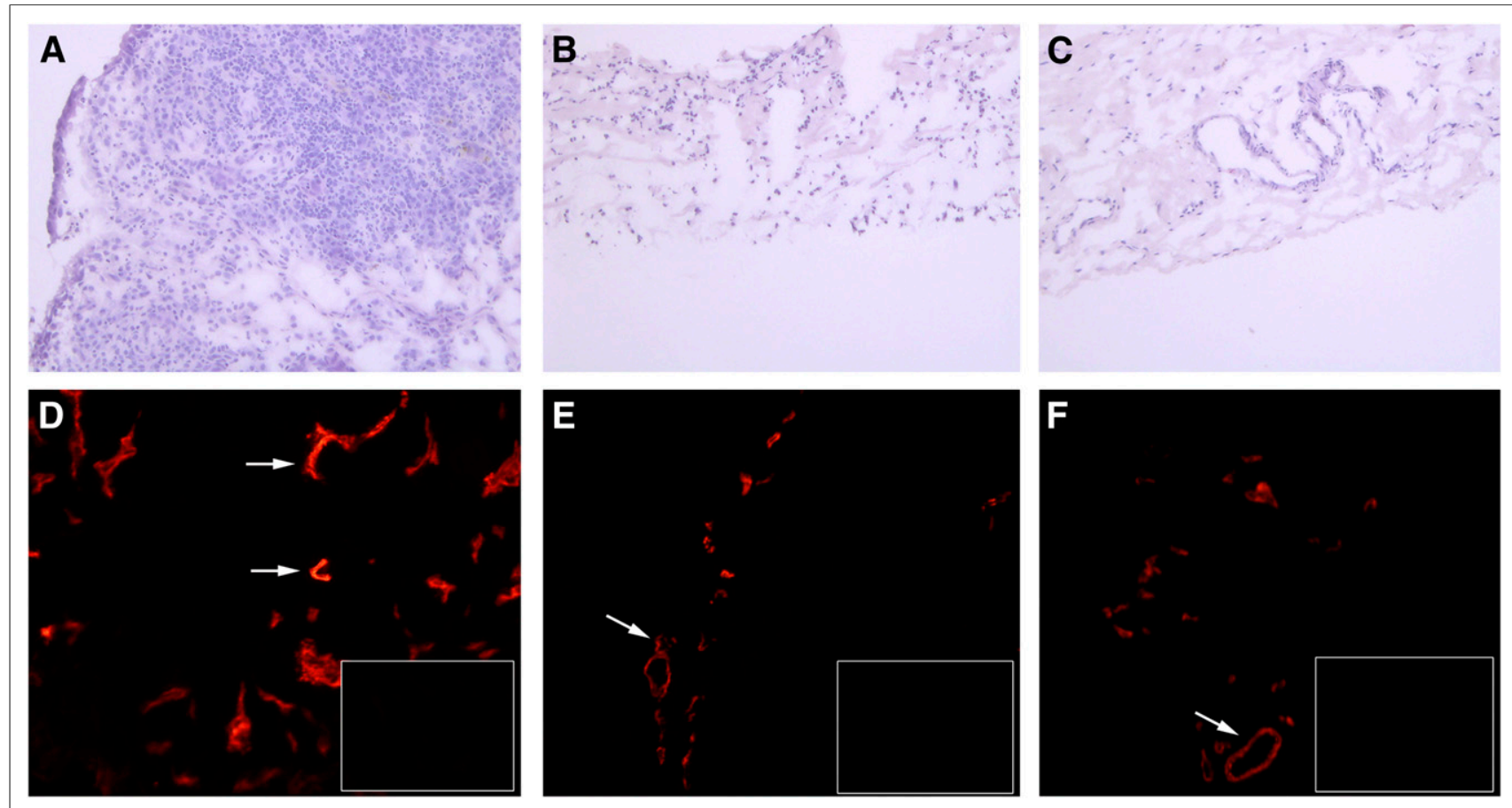

FIGURE 1. Induction of VAP-1 in rabbit and human synovial inflammation. (A-C) Hematoxylin-eosin stainings of inflamed human synovium (A), inflamed rabbit synovium (B), and synovium of control knee of rabbit (C). (D-F) Immunohistochemical stainings of VAP-1 in inflamed human synovium (D), inflamed rabbit synovium (E), and synovium of control knee of rabbit (F). Insets show stainings with control antibody. Some positive (red) vessels are indicated by arrows. Magnification is $\times 100$. 


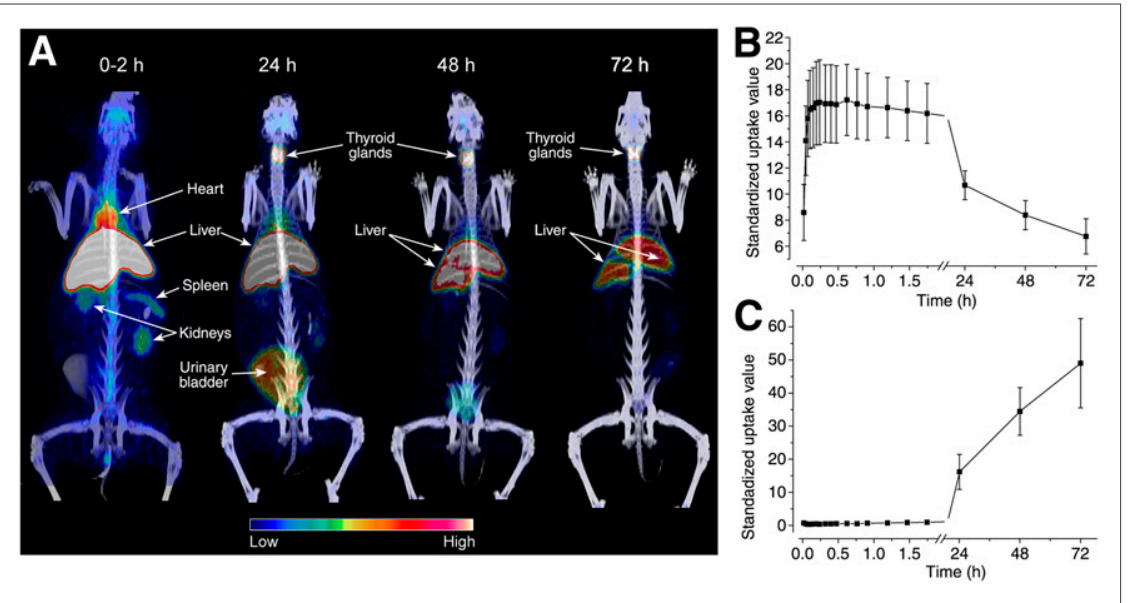

FIGURE 2. Whole-body distribution of ${ }^{124}$ I-BTT-1023 in healthy rabbit. (A) PET/CT images showing distribution of radioactivity up to $72 \mathrm{~h}$ after injection. (B and $\mathrm{C}$ ) Time-activity curves of liver (B) and thyroid glands (C).

antibody (Supplemental Fig. 1A). Also, in flow cytometric analyses, $100 \%$ of human VAP-1-transfected cells had positive ${ }^{123} \mathrm{I}-$ BTT-1023 staining, whereas mock-transfected cells remained negative (Supplemental Fig. 1B).

\section{PET Studies}

Representative PET/CT images and time-activity curves of liver and thyroid glands are presented in Figure 2 and Supplemental Table 1 . Radioactivity accumulated in the liver rapidly after injection and declined slowly during the 4-d experiment. The thyroids showed high uptake at day 4 , which was probably due to the deiodination of the antibody. Radioactivity cleared rapidly from the heart. Only a small amount of radioactivity was present in the kidneys, which function as an elimination route for free iodine.

Ex vivo measurements confirmed the in vivo imaging results at $72 \mathrm{~h}$ after injection (Supplemental Table 2; Supplemental Fig. 2). The highest ${ }^{124}$ I-radioactivity concentrations were in the thyroid and the liver. All other organs had a radioactivity concentration below 0.1 percentage injected radioactivity per gram of tissue. The brain had the lowest uptake. Linear regression analysis showed a strong correlation between the radioactivity of tissue samples and PET values $(R=0.94 ; P<0.0001)$ (Supplemental Fig. 2B).
In small-intestine cryosections, the radioactivity concentrated in the smooth muscle rather than in the lining or mucosal part of the intestine. These observations are in line with anti-VAP-1 immunohistochemistry, which demonstrates expression of VAP-1 both in the smooth muscle cell layer and in the subpopulation of mucosal vessels (Figs. 3A-3C). According to the autoradiography of small-intestine cryosections, uptake of ${ }^{124}$ I-BTT-1023 was significantly higher in VAP-1-positive areas than in VAP-1-negative areas, at $4.8 \pm 2.1$ and $0.93 \pm 0.61$ photostimulated luminescence per square millimeter, respectively $(P<0.001$; Fig. 3D).

The human radiation dosimetry estimates extrapolated from the rabbit data are presented in Table 1. The mean effective dose due to ${ }^{124}$ I-BTT-1023 was 0.55 $\mathrm{mSv} / \mathrm{MBq}$, if one assumes blockage of thyroid uptake. Importantly, only minimal radioactivity was found in bone marrow.

Further details on the results are provided as supplemental data.

\section{SPECT/CT}

Inflamed rabbit knee visualized with SPECT/CT had inflammation-to-control joint and inflammation-to-muscle ratios of 1.2 \pm 0.1 and $2.2 \pm 1.0$, respectively (Fig. 4). In vivo SPECT results were confirmed by ex vivo measurements demonstrating inflammation-to-control joint and inflammation-to-muscle ratios of 1.7 \pm 0.5 and $3.3 \pm 2.4$, respectively.

\section{DISCUSSION}

We demonstrated that the fully human antibody BTT-1023 can be labeled with both ${ }^{124} \mathrm{I}$ and ${ }^{123} \mathrm{I}$ without eliminating its affinity for human VAP-1. The whole-body distribution and pharmacokinetics of intravenous ${ }^{124}$ I-BTT-1023 were elucidated by PET/CT of healthy rabbits. In addition, we showed that ${ }^{123}$ I-BTT-1023 SPECT/CT can detect inflammation in a rabbit model of arthritis.

The high liver uptake is probably mediated in large part by VAP-1, since the antigen is found on the sinusoidal endothelia (12). However, a minor part of the signal is likely caused by the

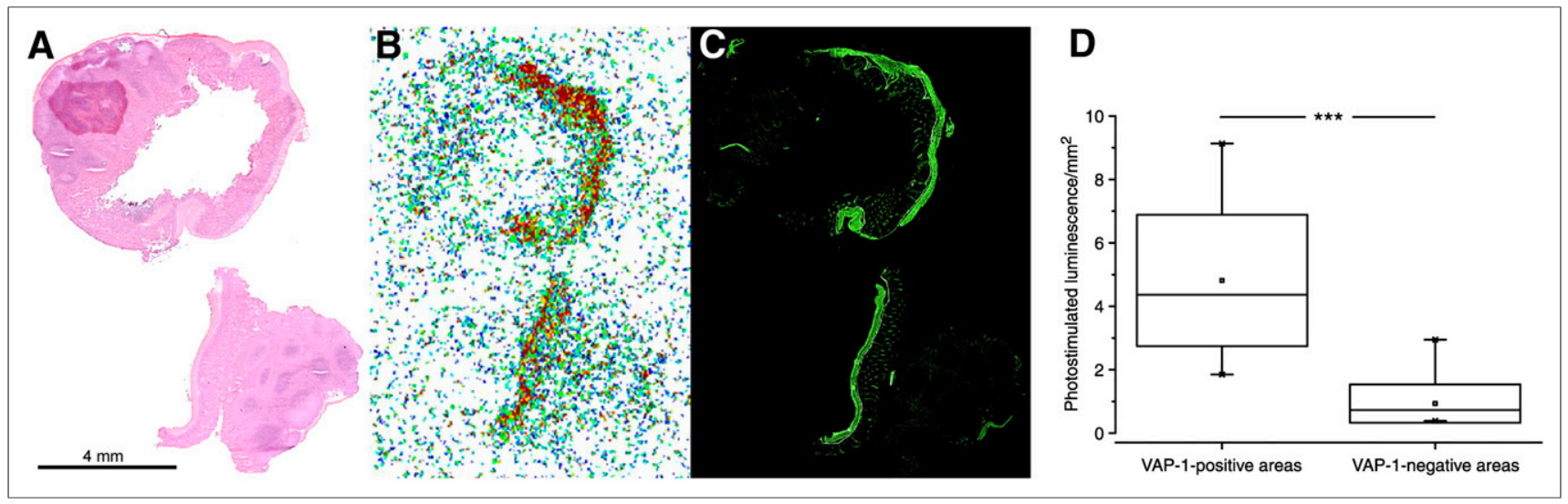

FIGURE 3. Binding of ${ }^{124}$ I-BTT-1023 to VAP-1-positive vessels in small intestine. (A-C) Hematoxylin-eosin staining (A), autoradiograph (B), and anti-VAP-1 immunohistochemical staining (C) of small-intestine cryosections. (D) Distribution of radioactivity at $72 \mathrm{~h}$ after injection in VAP-1-negative and VAP-1-positive areas of small intestine (Student $t$ test). 
TABLE 1

Human Radiation Dosimetry Extrapolated from Rabbit ${ }^{124}$ I-BTT-1023 Data

\begin{tabular}{lrr}
\hline \multicolumn{1}{c}{ Organ } & NND $(\mathrm{h})$ & Dose $(\mathrm{mSv} / \mathrm{MBq})$ \\
\hline Brain & $0.41 \pm 0.07$ & $0.15 \pm 0.03$ \\
Heart wall & $1.10 \pm 0.33$ & $0.88 \pm 0.21$ \\
Kidneys & $0.36 \pm 0.14$ & $0.52 \pm 0.13$ \\
Liver & $27.00 \pm 9.90$ & $3.20 \pm 1.10$ \\
Lungs & $5.40 \pm 2.20$ & $1.10 \pm 0.34$ \\
Spleen & $0.31 \pm 0.21$ & $0.47 \pm 0.20$ \\
Stomach + content (wall for dose) & $0.16 \pm 0.05$ & $0.36 \pm 0.05$ \\
Thyroid gland & $9.90 \pm 3.50$ & $63.00 \pm 22.00$ \\
Urinary bladder + content (wall for dose) & $0.93 \pm 0.15$ & $0.59 \pm 0.06$ \\
Remainder of body & $33.00 \pm 14.00$ & $3.70 \pm 1.10$ \\
Effective dose & & $0.55 \pm 0.08$ \\
Effective dose without thyroid gland uptake & & \\
& & \\
\hline Results are expressed as mean \pm SD $(n=10)$. NND $=$ normalized number of disintegrations. & \\
\hline
\end{tabular}

hepatic elimination of the antibody. Previously, Jaakkola et al. studied the in vivo behavior of ${ }^{123}$ I-labeled chimeric mouse-human anti-VAP-1 antibody in dogs and pigs using SPECT (11). Their results suggest that uptake in liver is VAP-1-specific. Imaging of inflammation in the abdominal area is limited, since the liver shows high uptake. Thyroid radioactivity is most plausibly due to the deiodination of ${ }^{124}$ I from the antibody. Free iodine is rapidly taken up by thyroid glands, and the excess is excreted via the kidneys to the urine.

In vivo imaging of synovial inflammation was demonstrated in an animal model of mild arthritis. Although the difference between affected and control joints was modest, similar ratios have been found with ${ }^{18} \mathrm{~F}-\mathrm{FDG}$ in the same animal model (Autio et al., unpublished data, 2012). The current detection of even very mild inflammation indicates the imaging potential of this tracer. The expression of VAP-1 and its regulation are not identical in humans and rabbits. Intravenously injected antibody detects VAP-1 also in the control knee of rabbits, and rabbit heart endothelial cell cultures express VAP-1 on the surface, whereas in humans the surface expression of endothelial VAP-1 is not detectable in noninflamed skin and human endothelial cell cultures do not express VAP-1 on the surface $(6,13)$.

The estimated mean effective dose for a $70-\mathrm{kg}$ man resulting from ${ }^{124} \mathrm{I}$-BTT-1023 (3.66 mSv/MBq) is rather high. However, with effective blocking of the thyroid gland by an adequate regimen, the estimated human radiation dose would decrease to a more acceptable level (0.55 mSv/MBq). Clinical PET imaging and dose

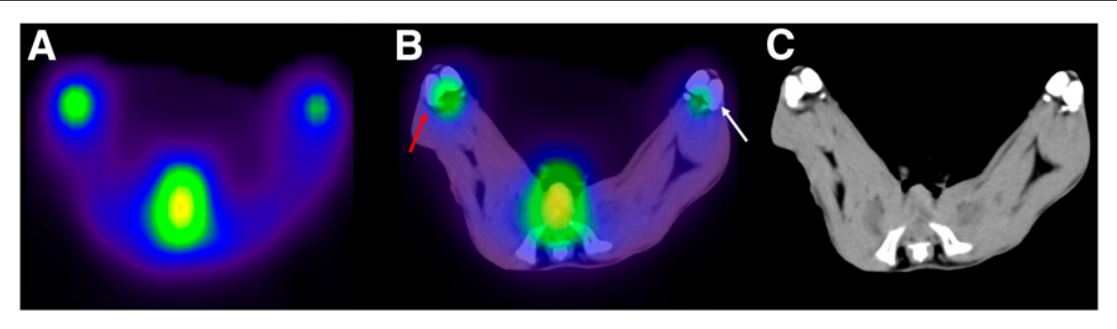

FIGURE 4. ${ }^{123} \mid-B T T-1023$ detection of synovial inflammation in rabbit knee. Transaxial SPECT (A), SPECT/CT (B), and CT (C) images show localization of radioactivity in inflamed knee (red arrow) and control knee (white arrow) at $2 \mathrm{~h}$ after injection. estimations of several ${ }^{124}$ I-labeled substances have been reported by other researchers (14-18).

To obtain proper PET images, the injected radioactivity of ${ }^{124} \mathrm{I}$ would ideally need to be higher than that of other PET radionuclides since only $25 \%$ of the ${ }^{124}$ I decay is by positron emission, with the rest being $\gamma$ - and $x$-rays. On the other hand, the longer physical half-life of ${ }^{124}$ I facilitates longer acquisition of PET data, improving the image quality. Detection of ${ }^{124}$ I-BTT-1023for example, in an inflamed human joint—should be possible with injected radioactivity of $0.5-0.7 \mathrm{MBq} / \mathrm{kg}$ since there is less background radioactivity, scatter, and random radioactivity in the vicinity. Moreover, the joint is reasonably easy to stabilize to minimize signal noise.

In general, imaging agents targeting VAP-1 could be valuable not only for the diagnosis and planning of treatment for patients with inflammatory conditions, such as rheumatoid arthritis, but also for the drug discovery and development processes. In addition to therapy monitoring, VAP-1 imaging would provide a scientific tool for in vivo imaging of leukocyte trafficking at the sites of inflammation. In vivo imaging of inflammation using VAP-1 as a target molecule and radiolabeled BTT-1023 as a tracer, such as in patients with rheumatoid arthritis, could help in stratifying patients for BTT-1023 antiinflammatory treatment.

\section{CONCLUSION}

BTT-1023 retained its biologic activity to bind VAP-1 after radioiodination in both in vitro and in vivo settings, and PET/CT imaging elucidated the whole-body distribution and pharmacokinetics over $4 \mathrm{~d}$ in rabbits. Most importantly, radiolabeled BTT-1023 was able to visualize mildly inflamed rabbit joints in SPECT/CT. There is a need for further studies with inflammation models to validate its future use in different clinical settings.

\section{DISCLOSURE}

The costs of publication of this article were defrayed in part by the payment of page charges. Therefore, and solely to 
indicate this fact, this article is hereby marked "advertisement" in accordance with 18 USC section 1734 . The study was conducted within the Finnish Center of Excellence in Molecular Imaging in Cardiovascular and Metabolic Research supported by the Academy of Finland, University of Turku, Turku University Hospital, and Åbo Akademi University. Petri J. Vainio and Jani Vainio are employees of Biotie Therapies Corp. Antti Mali is an employee of MAP Medical Technologies. No other potential conflict of interest relevant to this article was reported.

\section{ACKNOWLEDGMENTS}

English language proofreading of this article was kindly performed by Robert M. Badeau, PhD. Tuomo Nikula, Heidi Liljenbäck, and Erica Nyman are acknowledged for technical assistance.

\section{REFERENCES}

1. Salmi M, Jalkanen S. A 90-kilodalton endothelial cell molecule mediating lymphocyte binding in humans. Science. 1992;257:1407-1409.

2. Salmi M, Tohka S, Berg EL, Butcher EC, Jalkanen S. Vascular adhesion protein 1 (VAP-1) mediates lymphocyte subtype-specific, selectin-independent recognition of vascular endothelium in human lymph nodes. J Exp Med. 1997;186:589-600.

3. Merinen M, Irjala H, Salmi M, Jaakkola I, Hänninen A, Jalkanen S. Vascular adhesion protein-1 is involved in both acute and chronic inflammation in the mouse. Am J Pathol. 2005;166:793-800.

4. Salmi M, Kalimo K, Jalkanen S. Induction and function of vascular adhesion protein-1 at sites of inflammation. J Exp Med. 1993;178:2255-2260.

5. Marttila-Ichihara F, Smith DJ, Stolen C, et al. Vascular amine oxidases are needed for leukocyte extravasation into inflamed joints in vivo. Arthritis Rheum. 2006;54:2852-2862.
6. Salmi M, Yegutkin GG, Lehvonen R, Koskinen K, Salminen T, Jalkanen S. A cell surface amine oxidase directly controls lymphocyte migration. Immunity. 2001;14:265-276.

7. Madej A, Reich A, Orda A, Szepietowski JC. Vascular adhesion protein-1 (VAP-1) is overexpressed in psoriatic patients. J Eur Acad Dermatol Venereol. 2007;21:72-78.

8. Kirton CM, Laukkanen ML, Nieminen A, et al. Function-blocking antibodies to human vascular adhesion protein-1: a potential anti-inflammatory therapy. Eur $J$ Immunol. 2005;35:3119-3130.

9. Boerman OC, Oyen WJ. Immuno-PET of cancer: a revival of antibody imaging. J Nucl Med. 2011;52:1171-1172.

10. Mikkola J, Smith D, Vainio J, Vainio P, Vuorio P, inventors. Biotie Therapies Corporation, assignee. Novel fully human anti-vap-1 monoclonal antibodies. Patent WO2008129124 A1. October 30, 2008.

11. Jaakkola K, Nikula T, Holopainen R, et al. In vivo detection of vascular adhesion protein-1 in experimental inflammation. Am J Pathol. 2000;157:463-471.

12. Lalor PF, Edwards S, McNab G, Salmi M, Jalkanen S, Adams D. Vascular adhesion protein-1 mediates adhesion and transmigration of lymphocytes on human hepatic endothelial cells. J Immunol. 2002;169:983-992.

13. Vainio PJ, Kortekangas-Savolainen O, Mikkola JH, et al. Safety of blocking vascular adhesion protein-1 in patients with contact dermatitis. Basic Clin Pharmacol Toxicol. 2005;96:429-435.

14. Tran L, Vogel WV, Sinaasappel M, et al. The pharmacokinetics of ${ }^{124}$ I-rituximab in patients with rheumatoid arthritis. Hum Antibodies. 2011;20:7-14.

15. Jayson GC, Zweit J, Jackson A, et al. Molecular imaging and biological evaluation of HuMV833 anti-VEGF antibody: implications for trial design of antiangiogenic antibodies. J Natl Cancer Inst. 2002;94:1484-1493.

16. Farmakis G, Brandau W, Hellwig D, et al. PET imaging with p-[I-124]iodo-1phenylalanine as a new tool for diagnosis and postoperative control in patients with glioma. Clin Nucl Med. 2008;33:441-442.

17. Divgi CR, Pandit-Taskar N, Jungbluth AA, et al. Preoperative characterisation of clear-cell renal carcinoma using iodine-124-labelled antibody chimeric G250 $\left.{ }^{124} \mathrm{I}-\mathrm{cG} 250\right)$ and PET in patients with renal masses: a phase I trial. Lancet Oncol. 2007;8:304-310.

18. Carrasquillo JA, Pandit-Taskar N, O'Donoghue JA, et al. ${ }^{124}$ I-huA33 antibody PET of colorectal cancer. J Nucl Med. 2011;52:1173-1180. 